\title{
A Graph-Temporal fused dual-input Convolutional Neural Network for Detecting Sleep Stages from EEG Signals
}

\author{
Qing Cai, Zhongke Gao, Senior Member, IEEE, Jianpeng An, Shuang Gao, Celso Grebogi
}

\begin{abstract}
Sleep is an essential integrant in everyone's daily life. Thereby, it is an important but challenging problem to construct a reliable and stable system, that can monitor user's sleep quality automatically. In this work, we combine complex network and deep learning to propose a novel Graph-Temporal fused dual-input Convolutional Neural Network (CNN) method to detect sleep stages by using the Sleep-EDF database. Firstly, we segment each single-channel EEG signal into non-overlapping 30s epochs to set up the network. For that, we map each epoch into a Limited Penetrable Visibility Graph (LPVG) and obtain the corresponding Degree Sequence (DS) by calculating the node degree. Finally, the DSs and the 30s EEG epochs are combined as inputs of the novel Graph-Temporal fused dual-input CNN to learn about the graph topology and about the temporal feature representations of the raw data for the purpose of classifying the sleep stages into the two-, three-, four-, five-, and six-state. Notably, the classification accuracy of six-state stage detection is $87.21 \%$ and the corresponding Kappa value is 0.80 . The results demonstrate the effectiveness of our model structure in detecting sleep states, whereby they further provide a basic strategy for future sleep research.
\end{abstract}

Index Terms-Sleep stage detection; Limited Penetrable Visibility Graph(LPVG); Complex network; electroencephalogram (EEG); Convolutional neural network (CNN)

\section{INTRODUCTION}

$\mathbf{S}$ LEEP plays a crucial part in everyone's daily life wellbeing as it is strongly associated with performance of many essential daily activities, such as reasoning, working efficiency, and mental concentration. Generally speaking, there are six sleep stages, wake-fullness (W), non-rapid eye movement (stages 1-4, from light to deep sleep), and rapid eye movement (REM) in a sleeping cycle. Sleep is vital for the body and brain recover from fatigue while cycling through the sleep stages [1]. However, with increasing life pressure, a larger number of people are afflicted with sleep-related disorders and diseases. Sleep disorders are significant risk factors leading to neurological diseases, impaired performance, and decreased quality of life. The investigation of sleep disorders has become a topic of great importance in recent years.

This work was supported by the National Natural Science Foundation of China under Grant Nos. 61922062, and 61873181. (Corresponding author: Zhongke Gao)

Z. Gao Q. Cai and J. An are with the School of Electrical and Information Engineering, Tianjin University, Tianjin 300072, China (e-mails: zhongkegao@tju.edu.cn).

S. Gao is with First Teaching Hospital of Tianjin University of Traditional Chinese Medicine, Tianjin 300193, China.

C. Grebogi is with Institute for Complex Systems and Mathematical Biology, King's College, University of Aberdeen, Aberdeen AB24 3UE, UK.
Polysomnographic recordings, which are generally composed of EEG, EOG, ECG, and EMG, are acquired from patients to carry out the investigations. The polysomnographic recordings are segmented into 30s epochs and classified into the six stages by a disciplined expert in compliance with the Rechtschaffen \& Kales $(\mathrm{R} \& \mathrm{~K})$ rules [2]. This process is called sleep stage detection or sleep stage scoring, which is an essential and indispensable procedure of sleep-related disorder diagnosis, sleep-related disease treatment, and sleep research. However, analyzing polysomnographic recordings manually is laborexhaustive, time-consuming and subject to experts mental state. Developing an automatic sleep stage detection system, which can provide an objective and repeatable sleep stage classification results, is in urgent need.

A number of methods using physiological signals have been proposed to detect sleep stages including support vector machine recursive feature elimination system [3], entropy features extracted from five frequency bands [4], wavelet coefficients entropy [5], recurrence quantification analysis [6], time-domain features as well as the structural graph similarity [7] and difference visibility graph [8]. The above methods extract time-domain, frequency-domain and non-linear features from each recording epoch. The features are then selected to realize an optimal combination for sleep stage detection.

Despite the effectiveness of sleep stage detection, the above methods are task-specific and limited by the features that are manually selected by the researchers. With the goal of analyzing changing, there is a need to reconsider the best suited features. Fortunately, deep learning can be employed to address the problem as it is an approach that can extract the significant features directly from the data and, in addition, eliminate the need for manual feature extraction. With the rapid development of some basic but specific neural networks, including the Convolutional Neural Network (CNN) and LongShort Term Memory (LSTM), better and deeper learning approaches have been applied in the analysis of physiological signals. For example, driver fatigue detection [9], ECG classification [10], SSMVEP-based BCI investigation [11] and sleep stage detection [12-16].

In past decades, the complex network theory has emerged and contributed significantly for the characterization of complex systems. Notably, the complex network analysis of time series has achieved impressive advances in diverse research areas by investigating the dynamical behaviors underlying the time series. References [17] and [18] presented reviews of the rapid development in complex network-based time series 
analysis. Among the published complex network methods, the Visibility Graph (VG) proposed by Lacasa et al. [19], has established itself an efficient graph construction approach in the analysis of EEG signals [20], human heartbeat dynamics [21], crude oil price [22], and others. Built on the basis of the computationally efficient and analytically tractable VG method, we proposed a LPVG method [23], which not only possesses the advantages of visibility graph but also show stronger noise resistance.

The above deep learning methods, using single-channel information derived from raw polysomnographic recordings, fulfills the task of sleep stage detection. Additionally, the LPVG method has been applied to time series analysis from graph theory [24-26]. In this work, we combine the LPVG and CNN to detect the sleep stages from a single-channel EEG signal. Each EEG epoch corresponding to one sleep stage is converted into a LPVG. Then the degree sequences of the LPVGs and the raw EEG epochs are evaluated by a novel dualinput CNN for sleep stage detection. By this way, we can fuse both the temporal information and the graph representation to improve the detection accuracy of sleep stages.

\section{EEG DATASET}

The sleep EEG dataset analyzed in this paper, which is used to demonstrate the efficiency of the proposed methodology, is taken from the publicly available Physionet's Sleep-EDF databank [27]. This dataset includes 61 polysomnographic recordings with the corresponding sleep stage annotations. The dataset comprises two groups: the Sleep Cassette group composed by healthy volunteers and the Sleep Telemetry group composed by subjects suffering from mild sleep disturbance. We use 10 subjects from the SC group and 10 subjects from the ST group in this work. For each subject, their respective polysomnographic recordings consisting of one EOG channel and two EEG channels (from $\mathrm{Fpz}-\mathrm{Cz}$ and $\mathrm{Pz}-\mathrm{Oz}$ electrodes location), are saved in a corresponding EDF-File. The three channel signals are obtained with a sampling frequency of $100 \mathrm{~Hz}$. Each 30s EEG epoch, containing 3000 data points, is categorised as one of six sleep stages based on R\&K rules by well-trained experts. The six sleep stages include awake (W), stage 1 (S1), stage 2 (S2), stage 3 (S3), stage 4 (S4), and rapid eye movement (REM). In this work, we perform six-state classification (W, S1, S2, S3, S4, REM); five-state classification (W, S1, S2, S3\&S4, REM); four-state classification (W, S1\&S2, S3\&S4, REM); three-state classification (W, S1-S4, REM); two-state classification (W, S1-S4\&REM). We employ the Fpz-Cz channel to evaluate performances of our proposed Graph-Temporal fused dual-input CNN system. We show the number distribution of epochs for each of the six sleep stages in Tab. I.

TABLE I

THE NUMBER OF EPOCHS OF THE SIX SLEEP STAGES.

\begin{tabular}{|c|c|c|c|c|c|c|}
\hline Sleep stage & W & S1 & S2 & S3 & S4 & REM \\
\hline Epoch number & 19658 & 1830 & 8816 & 1716 & 1431 & 3775 \\
\hline
\end{tabular}

\section{METHODOLOGY}

In this section, we firstly introduce the construction of LPVG for each EEG epoch, then derive the degree sequence from LPVG and employ it as the graph representation. At last, we present the developed framework, graph-temporal fused dual-input convolutional neural network, with model structure and implementation details.

\section{A. Limited Penetrable Visibility Graph and Degree Sequence}

Suppose $S=\left\{s_{1}, s_{2}, s_{3} \ldots s_{N}\right\}$ denotes one of the EEG epochs, $s_{i}$ represents the value of the $i_{t h}$ point in the EEG epoch $\mathrm{S}$. We display the EEG epoch in the shape of vertical bars, whose heights correspond to the values of time points and whose positions are the same as in the time series, as shown in Fig. 1. We link vertical bars pairwise if the straight visibility line is not hampered by any intermediate vertical bars, so there exists a coupling between the two bars, or the two nodes $\left(a_{i j}=1\right.$ for nodes $s_{i}$ and $\left.s_{j}\right)$. Otherwise, the two nodes are uncoupled $\left(a_{i j}=0\right.$ for nodes $s_{i}$ and $\left.s_{j}\right)$. Formally, we establish the algorithm principle of VG: two arbitrary nodes $s_{i}$ and $s_{j}$ become coupled by a visibility line in the associated visibility graph, if any other data $s_{k}$ placed between them fulfills:

$$
s_{k}<s_{i}+\left(s_{j}-s_{i}\right) \frac{k-i}{j-i}
$$

In this work, we develop LPVG by adding a limited penetrable distance on the basis of VG. If we set the limited penetrable distance to be $L$, two nodes are coupled if the number of intermediate nodes that blocks the visibility line is no more than $L$. In Fig. 1, we illustrate a LPVG with the limited penetrable distance $L$ being 1 .

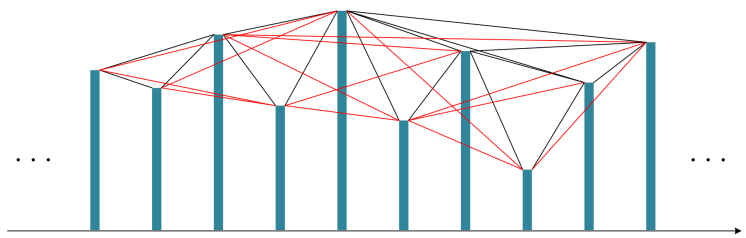

(a)

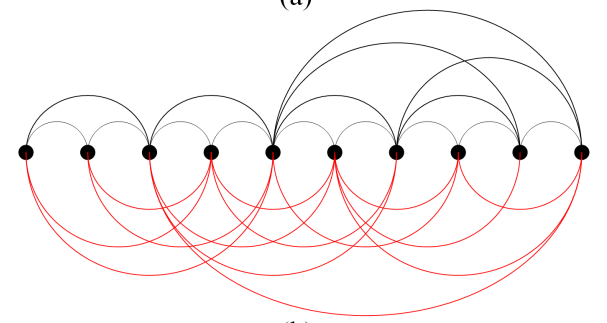

(b)

Fig. 1. Illustration of (a) an EEG epoch and (b) its corresponding LPVG with the limited penetrable distance $L$ being 1 . The black lines represent the visibility lines in VG, while the red lines represent the added visibility lines in LPVG. The visibility lines between data points define the edges coupling the nodes in the graph.

As shown in Fig.1, we carry out the above procedure and derive a series of LPVGs corresponding to different sleep stage epochs. The degree sequence is highly informative on 
the associated dynamical process, working even better than more sophisticated topological metrics [28]-[29]. We extract the Degree Sequence (DS) $\left\{K_{i}=\sum_{j=1, j \neq i}^{N} a_{i j}\right\}_{i=1}^{N}$ so as to characterize the complexity and the dynamics of the EEG signals.

\section{B. Dual-input CNN Structure}

We design a paralleled dual-input CNN structure to fuse the temporal information from the raw EEG signal and the graph representation from the constructed LPVG, as shown in Fig. 2.

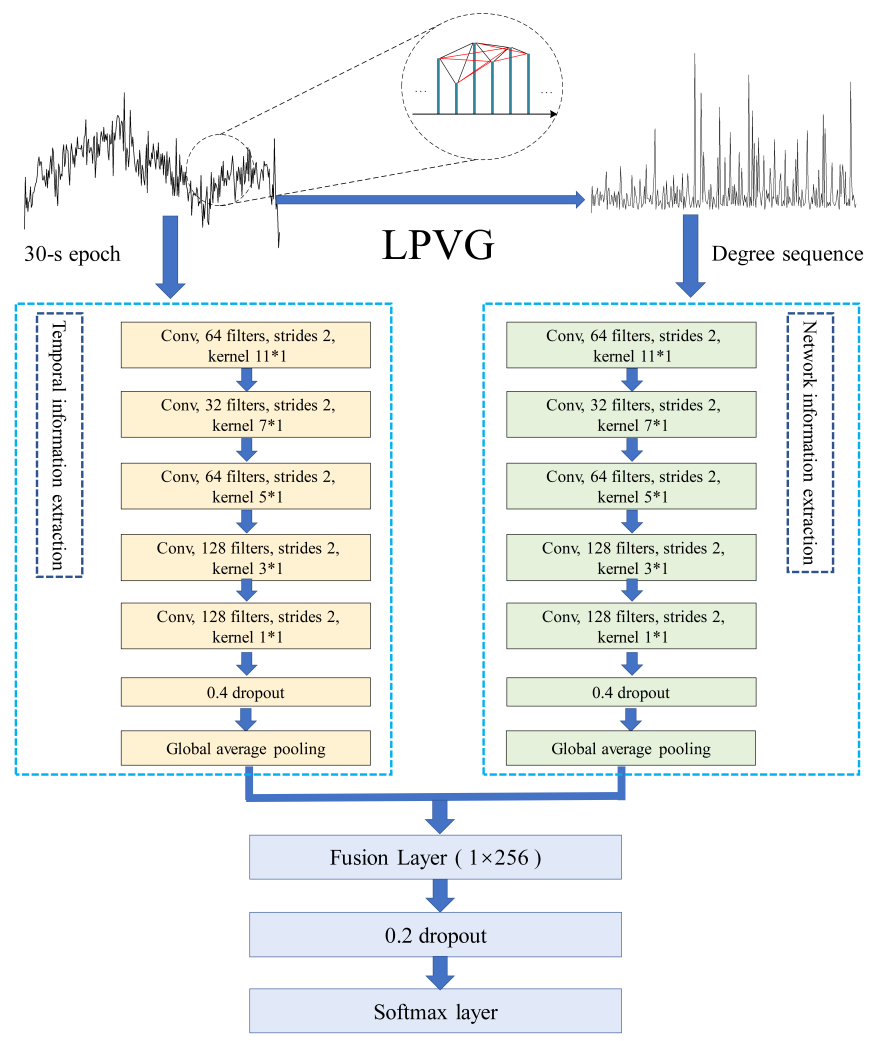

Fig. 2. The framework of the novel graph-temporal fused dual-input CNN sleep stage detection system.

The dual inputs used in our structure are raw EEG epochs and DSs, both experiencing the five convolutional layers. Five kernels with size $11 * 1,7 * 1,5 * 1,3 * 1$ and $1 *$ 1 are chosen for the five convolutional layers, respectively. In addition, the number of filters in the convolutional layers are $64,32,64,128$ and 128, respectively. There are three operations, 1D-convolution with its filters, batch normalization [30] and Rectified Linear Unit (ReLU) activation [31], carried out successively in each convolutional layer. The batch normalization and ReLU activation constitute an activation block to bypass the normalization operation on input data.

After the five convolutional layers, we employ a global average pooling. After the convolution and pooling operations, we then concatenate and fuse the two outputs to go through a dropout layer and a softmax layer. Finally, cross-entropy objective function is used in the softmax layer to produce a probability for each sleep stage. Thus, the stage, which obtains the maximum probability, is regarded as the predicted annotation. Through five convolution operations, the two inputs are processed layer by layer to yield better representations in order to obtain the following sleep stage detection. The fusion layer integrates and fuses the features from the two inputs and conduct the detection of sleep states.

Furthermore, the Glorot normal initializer is applied during the model training process to initialize the weights of the convolutional layers. The Adam optimizer is employed during the model training process with learning rate $0.0001, \beta_{1}=0.9$ and $\beta_{2}=0.999$ to optimize the model. $L 2$ regularization is applied in each convolutional layer with $l=0.0001$. The number of learning iterations is set at 300 and the batch size at 256. In the case that the validation accuracy stops increasing, the patience of early stop scheme is set as 30 with the aim to avoid overfitting.

\section{RESUlTS}

We employ 10-fold cross validation strategy to demonstrate the effectiveness of the graph-temporal fused dual-input CNN method. We randomly divide the whole sleep dataset into 10 subsets, then we perform validation by selecting 1 subset as the testing set while the remaining 9 subsets as the training set. This procedure is repeated 10 times such that each of the 10 subsets can be enumerated as testing set. Thus, we obtain the average accuracy and the standard deviation of 10-fold validations. The Confusion Matrix (CM) across ten folds from the Graph-Temporal fused dual-input CNN system of two to six-state sleep stage detection are shown in Fig. 3. Based on each CM, we calculate the classification accuracies and Kappa to present the effectiveness of the Graph-Temporal fused dualinput CNN system. The detailed results of the two-state to six-state sleep stage detection are shown in Tab. II.

TABLE II

ACCURACY (\%) AND KAPPA VALUE RESULTS OF TWO-STATE TO SIX-STATE CLASSIFICATIONS OF FPZ-CZ

\begin{tabular}{|c|c|c|}
\hline Sleep state detection & Accucracy (\%) & Kappa value \\
\hline 2-state & $98.22 \pm 0.198$ & $0.9642 \pm 0.039$ \\
\hline 3-state & $94.14 \pm 0.263$ & $0.8977 \pm 0.061$ \\
\hline 4-state & $91.2 \pm 0.254$ & $0.8579 \pm 0.076$ \\
\hline 5-state & $89.1 \pm 0.41$ & $0.8294 \pm 0.017$ \\
\hline 6-state & $87.21 \pm 0.57$ & $0.7998 \pm 0.028$ \\
\hline
\end{tabular}

Sleep stage scoring, when performed manually by welltrained experts, is a laborious and time-consuming task. In this work, we develop a novel graph-temporal fused dualinput CNN method to realize sleep stage classification. The Sleep-EDF database has been used by many researchers to demonstrate the validity of their proposed sleep stage detection methods in literature. Tab. III presents the comparison of our proposed method with some other state-of-the-art studies using the Sleep-EDF database. The classification accuracy and Kappa results of 6-state detection are included. 

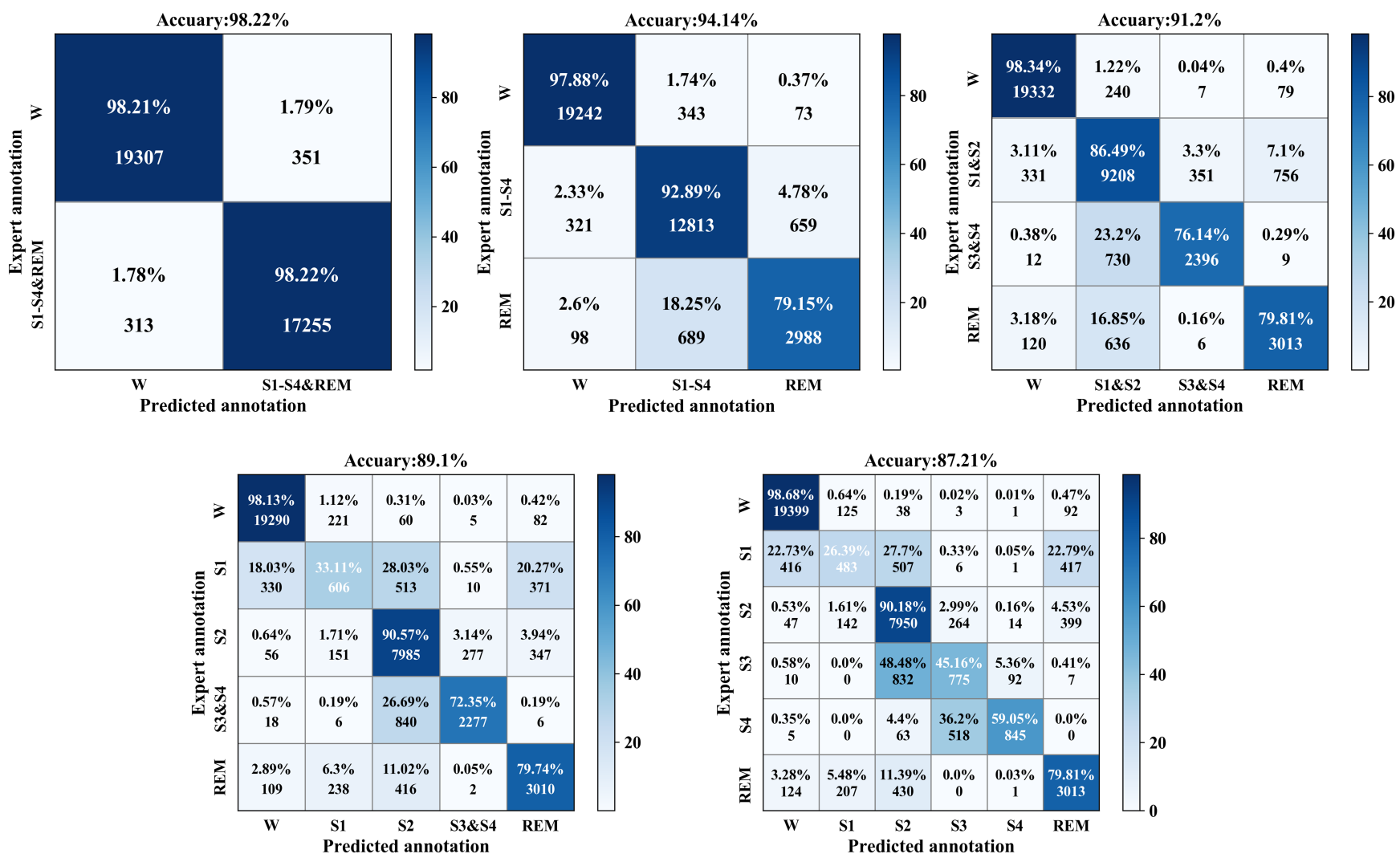

Fig. 3. The average CMs across ten folds of 2-state, 3-state, 4-state, 5-state, and 6-state sleep stage detection of Fpz-Cz channel.

\section{CONCLUSION}

In this work, we address the problem of detecting sleep stages automatically by combining complex network and deep learning. We propose a novel dual-input CNN method, which fuse graph representation from limited penetrable visibility graph and temporal information from raw EEG epoch. Firstly, we construct the LPVG associated with each sleep EEG epoch to extract the corresponding Degree Sequence (DS); secondly, the DSs and the 30s EEG epochs are fed into the novel dualinput to fuse temporal information and graph network features. Most existing methods require developers to have domain knowledge to extract features manually. However, the best suited features can often be hard to be identified. In this regard, our method is not limited to any domain knowledge. Our CNN structure allows the method to extract features automatically. More importantly, the Graph-Temporal fused dual-input CNN method presents a way of combing deep learning and complex network, which is very helpful for home sleep monitoring.

\section{REFERENCES}

[1] S. Mukherjee et al., "An Official American Thoracic Society Statement: The Importance of Healthy Sleep," Am. J. Resp. Crit. Care, vol. 191, no. 12, pp. 1450-1458, Jun. 2015.

[2] J. A. Hobson, "A Manual of Standardized Terminology, Techniques and Scoring System for Sleep Stages of Human Subjects.," Electroencephalogr. Clin. Neurophysiol., vol. 26, no. 6, p. 644, 1969.

[3] M. Adnane, Z. Jiang, and Z. Yan, "Sleep-wake stages classification and sleep efficiency estimation using single-lead electrocardiogram," Expert Syst. Appl., vol. 39, no. 1, pp. 1401-1413, Jan. 2012.
[4] Z. M. Zhang et al., "Efficient sleep classification based on entropy features and a support vector machine classifier," Physiol. Meas., Article vol. 39, no. 11, p. 13, Nov. 2018, Art. no. 115005.

[5] R. Boostani, F. Karimzadeh, and M. Nami, "A comparative review on sleep stage classification methods in patients and healthy individuals," Comput. Meth. Programs Biomed., Review vol. 140, pp. 77-91, Mar. 2017.

[6] U. R. Acharya, S. Bhat, O. Faust, H. Adeli, E. C. P. Chua, W. J. E. Lim, and J. E. W. Koh, "Nonlinear Dynamics Measures for Automated EEGBased Sleep Stage Detection," Eur. Neurol., Review vol. 74, no. 5-6, pp. 268-287, 2015

[7] M. Diykh, Y. Li, and P. Wen, "EEG Sleep Stages Classification Based on Time Domain Features and Structural Graph Similarity," IEEE Trans. Neural Syst. Rehabil. Eng., vol. 24, no. 11, pp. 1159-1168, Nov. 2016.

[8] G. Zhu, Y. Li, and P. Wen, "Analysis and Classification of Sleep Stages Based on Difference Visibility Graphs From a Single-Channel EEG Signal," IEEE J. Biomed. Health, vol. 18, no. 6, pp. 1813-1821, Nov. 2014.

[9] Z. Gao et al., "EEG-Based Spatio-Temporal Convolutional Neural Network for Driver Fatigue Evaluation," IEEE T. Neur. Lear., vol. 30, no. 9, pp. 2755-2763, Sep. 2019.

[10] S. M. Mathews, C. Kambhamettu, and K. E. Barner,"A novel application of deep learning for single-lead ECG classification," Comput. Biol. Med., Article vol. 99, pp. 53-62, Aug. 2018.

[11] Z. Gao et al., A deep learning method for improving the classification accuracy of SSMVEP-based BCI," IEEE Trans Circuits Syst II, to be published. DOI: 10.1109/TCSII.2020.2983389

[12] A. Sors, S. Bonnet, S. Mirek, L. Vercueil, and J. F. Payen, "A convolutional neural network for sleep stage scoring from raw singlechannel EEG," Biomed. Signal Proces., Article vol. 42, pp. 107-114, Apr. 2018.

[13] Z. H. Cui, X. W. Zheng, X. X. Shao, and L. Z. Cui, "Automatic Sleep Stage Classification Based on Convolutional Neural Network and FineGrained Segments," Complexity, 2018, Art. no. 9248410.

[14] A. H. Ansari et al., "Quiet sleep detection in preterm infants using deep convolutional neural networks," J. Neural Eng., Article vol. 15, no. 6, p. 11, Dec. 2018, Art. no. 066006. 
TABLE III

COMPARISON OF OTHER STATE-OF-THE-ART STUDIES USING SLEEP EDF DATASET.

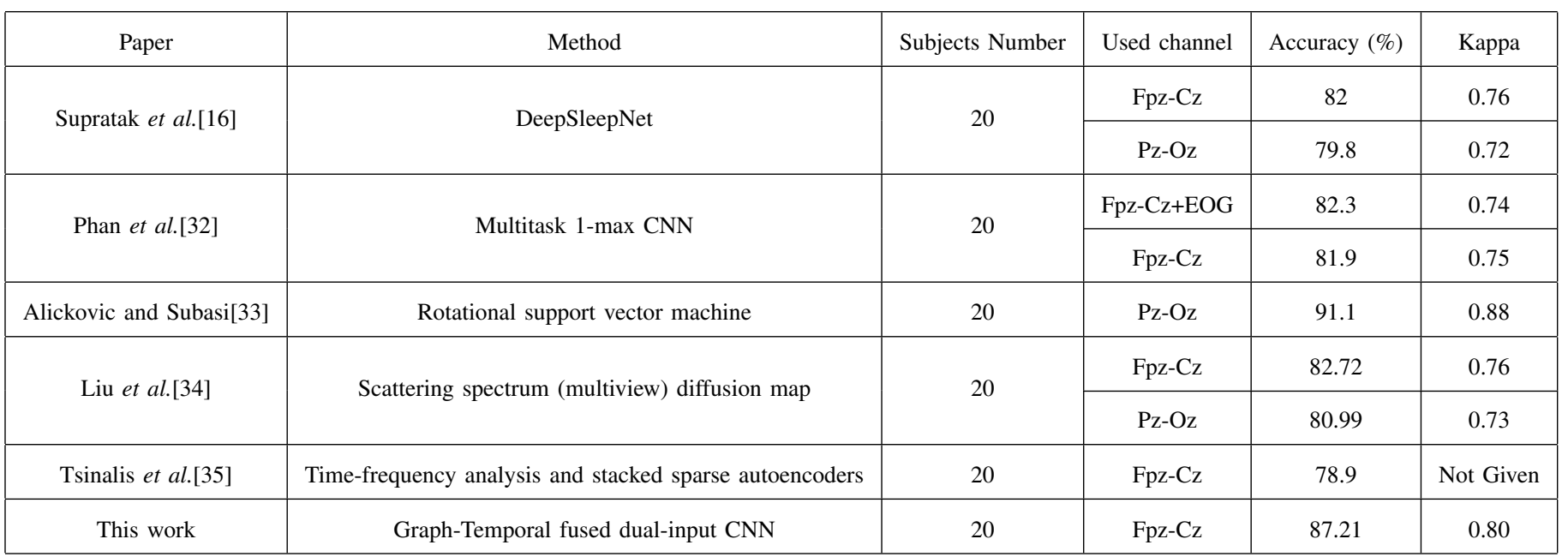

[15] N. Michielli, U. R. Acharya, and F. Molinari, "Cascaded LSTM recurrent neural network for automated sleep stage classification using singlechannel EEG signals," Comput. Biol. Med., vol. 106, pp. 71-81, Jan. 2019.

[16] A. Supratak, H. Dong, C. Wu, and Y. Guo, "DeepSleepNet: A Model for Automatic Sleep Stage Scoring Based on Raw Single-Channel EEG," IEEE Trans. Neural Syst. Rehabil. Eng., vol. 25, no. 11, pp. 1998-2008, Nov. 2017.

[17] Z. K. Gao, M. Small, and J. Kurths, "Complex network analysis of time series," EPL, Article vol. 116, no. 5, p. 7, Dec. 2016, Art. no. 50001.

[18] W. X. Wang, Y. C. Lai, and C. Grebogi, "Data based identification and prediction of nonlinear and complex dynamical systems," Phys. Rep., Review vol. 644, pp. 1-76, Jul. 2016.

[19] L. Lacasa, B. Luque, F. Ballesteros, J. Luque, and J. C. Nuno, "From time series to complex networks: The visibility graph," PNAS, Article vol. 105, no. 13, pp. 4972-4975, Apr. 2008.

[20] M. Ahmadlou, H. Adeli, and A. Adeli, "New diagnostic EEG markers of the Alzheimer's disease using visibility graph," J. Neural Transm., vol. 117, no. 9, pp. 1099-1109, Sep. 2010.

[21] Z. G. Shao, "Network analysis of human heartbeat dynamics," Appl. Phys. Lett., Article vol. 96, no. 7, p. 3, Feb. 2010, Art. no. 073703.

[22] H. Z. An, X. Y. Gao, W. Fang, X. Huang, and Y. H. Ding, "The role of fluctuating modes of autocorrelation in crude oil prices," Physica A, Article vol. 393, pp. 382-390, Jan. 2014.

[23] Z. K. Gao, Q. Cai, Y. X. Yang, W. D. Dang, and S. S. Zhang, ”Multiscale limited penetrable horizontal visibility graph for analyzing nonlinear time series," Sci. Rep., vol. 6, p. 35622, Oct. 2016.

[24] M. Wang, H. Xu, L. Tian, and H. E. Stanley, "Degree distributions and motif profiles of limited penetrable horizontal visibility graphs," Physica A, vol. 509, pp. 620-634, Nov. 2018.

[25] M. Wang et al., "Exact results of the limited penetrable horizontal visibility graph associated to random time series and its application," Sci. Rep., vol. 8, Mar 23. 2018, Art. no. 5130.

[26] X. Li, M. Sun, C. Gao, D. Han, and M. Wang, ”The parametric modified limited penetrable visibility graph for constructing complex networks from time series," Physica A, vol. 492, pp. 1097-1106, Feb. 2018.

[27] B. Kemp, A. H. Zwinderman, B. Tuk, H. A. C. Kamphuisen, and J. J. L. Oberye, "Analysis of a sleep-dependent neuronal feedback loop: The slow-wave microcontinuity of the EEG," IEEE T. Bio-Med Eng., vol. 47, no. 9 , pp. 1185-1194, Sep. 2000.

[28] B. Luque and L. Lacasa, "Canonical horizontal visibility graphs are uniquely determined by their degree sequence," Eur. Phys. J-Spec Top., vol. 226, no. 3, pp. 383-389, Feb. 2017.

[29] L. Lacasa, "On the degree distribution of horizontal visibility graphs associated with Markov processes and dynamical systems: diagrammatic and variational approaches," Nonlinearity, vol. 27, no. 9, pp. 2063-2093, Sep. 2014.

[30] S. Ioffe, and C. Szegedy, "Batch normalization: Accelerating deep network training by reducing internal covariate shift," in Proc. ICML32, Lille, France, 2015, pp. 448-456.

[31] V. Nair, and G. E. Hinton, Rectified linear units improve restricted boltzmann machines, in Proc. ICML27, Haifa, Israel, 2010, pp. 807-814.
[32] H. Phan, F. Andreotti, N. Cooray, O. Y. Chen, and M. De Vos, "Joint Classification and Prediction CNN Framework for Automatic Sleep Stage Classification," IEEE Trans. Biomed. Eng., vol. 66, no. 5, pp. 1285-1296, May. 2019.

[33] E. Alickovic and A. Subasi, "Ensemble SVM method for automatic sleep stage classification," IEEE Trans. Instrum.Meas., vol. 67, no. 6, pp. 1258C1265, Jun. 2018.

[34] G.R. Liu, Y. L. Lo, J. Malik, Y. C. Sheu, and H. T. Wu, "Diffuse to fuse EEG spectra C Intrinsic geometry of sleep dynamics for classification," Biomed. Signal Proces., vol. 55, Jan. 2020, Art. no.101576.

[35] O. Tsinalis, P. M. Matthews, and Y. K. Guo, "Automatic Sleep Stage Scoring Using Time-Frequency Analysis and Stacked Sparse Autoencoders," Ann. Biomed. Eng., Article vol. 44, no. 5, pp. 1587-1597, May. 2016. 